\title{
Microarrays and Surface Engineering for Bioanalysis
}

\author{
Hui Gao*, Sylvie Guinchard, François Crevoisier, Silvia Angeloni, and Hans Sigrist
}

\begin{abstract}
Molecular engineering and covalent immobilization of biomolecules on material surfaces whilst retaining specificity and biological functions is a major challenge in bioarray manufacturing and analysis. Photochemical functionalization of materials enables specific bioengineering and facile manufacturing of biotech products for microanalysis with concomitant surface passivation. Surfaces engineered with polysaccharides render materials hydrophilic, biocompatible and generally applicable for probe molecule immobilization. Products and procedures described support microanalytical investigations in all '-omics' domains.
\end{abstract}

Keywords: Functional platform $\cdot$ Microarray $\cdot$ Photoimmobilization $\cdot$ Proteomics $\cdot$ Surface passivation

\section{Introduction}

Bioarrays and biochips are emerging analytical tools for fast molecular screening, quality survey and control, and prognostic and diagnostic medical testing [1]. Molecular engineering and covalent immobilization of biomolecules on material surfaces retaining specificity and biological functions is a major challenge in bioarray manufacturing and analysis. Initiated by the pioneering work of Fodor and collaborators [2], microarray technology has developed into a useful platform technology with major applications in genomic research and development. More recently, the technology has expanded fast into the analytical investigation and characterization of proteins (proteomics), carbohydrate structures (glycomics) and the investigation of cell idiosyncrasies (cellomics). All '-omics' analytical domains deal with many probing sites or probe molecules on small analytical surfaces [3]. It is a challenging task to design

\footnotetext{
${ }^{*}$ Correspondence: Dr. H. Gao Centre Suisse d'Electronique et de Microtechnique SA CSEM SA

Jaquet-Droz 1 ,

CH-2007 Neuchâte

Tel.: +4132720 5177

Fax: +4132720 5750

E-Mail: hui.chaigao@csem.ch
}

and evaluate surfaces that are able to host many different types of biologically active probe molecules or cells. Microarrays, by definition, include many probe molecules, extracts or cells, all spotted on a carrier substrate. The number of immobilized molecular species may vary between tens to several thousands of different probe molecules within a few $\mathrm{cm}^{2}$, relating to low density and high density arrays, respectively.

Proteins, glycoconjugates, carbohydrates and cell systems demand specific immobilization processes for ultimate expression of their individual biological function. Whereas genomics requires immobilized polynucleotide probes capable of forming hydrogen bonds upon hybridization, successful surface bioengineering for proteomics and glycomics in contrast demands immobilization of proteins and glycans in their native 3-D folded, preferably nondenatured structures. Cell immobilization opts for surfaces that preserve full biological function of cell membrane constituents and the generation of an environment which mimics cell-cell contacts.

\section{Customized Bioengineering of Microarray Platforms}

In genechips, DNA and oligonucleotides, distinguished by their nucleotide sequence, optimally expose their hydrogen bonding valences for hybridization with complementary target polynucleotides. The major difference among individual DNA probe molecules is the number of hydrogen bonds that can be formed, or, due to a mismatch, are not formed with the analyte polynucleotides. Retention of 3-D structures is not required in DNA arrays and polynucleotide strand denaturation is imperative for successful hybridization. Preferred nucleotide probe molecule binding is effected with platforms providing the physical prerequisites for charge interaction (for instance aminopropylsilan, polylysine) or for covalent binding of amino-tailed probes (e.g. epoxysilane-, aldehyde platforms).

Arrays of peptides, proteins and glycoconjugates do not significantly differ from nucleotide arrays regarding the concept, the number of probes and detection of probe target or analyte interaction. In principle, some chemically engineered platforms used to date in genomic approaches are - in chemical and experimental terms - valid for proteomics and glycomics. However, the distinction is in the nature of the probe molecules to be immobilized, and the assay conditions used to effect analyte binding. An array of antibodies for instance necessitates retention of the structure of the antigen binding sites of all probe antibodies constituting the antibody array. Similarly, arrays of enzymes or receptor molecules demand active binding sites and binding assay conditions that confer with the probe's physical requirements $(\mathrm{pH}$, ionic strength, temperature, competitors) of all individual probing molecules. Membrane receptor molecules 
may further demand molecular amphipathy and orientation in order to express native biological functions.

In addition to satisfying the specific needs of '-omics' arrays, microarray technology has to overcome the phenomenon of non-specific binding: suppression of unspecific interaction of analyte components with probe sites other than the targeted site (specificity) and suppression of binding to the array surface within or surrounding individual spots (background). Microarraybased assay systems have to be constructed and optimized towards full probe-target binding avoiding all non-specific interactions. Denaturing conditions as used with DNA arrays cannot be applied in protein-protein or protein-carbohydrate binding assays. Structured proteins and carbohydrate binding proteins such as lectins are particularly sensitive to structural denaturation. Therefore, platform chemistries have to be tailored i) to match the chemical characteristics at the surface of the probe molecules (chemical needs), ii) to retain the probe's structure to express biological function (functional needs) and iii) to suppress non-specific adsorption of analyte constituents (assay needs). Arrays of cells request, in addition, conservation of cell vitality and the retention of concerted cellular functions (cell needs).

\section{Polysaccharide- and Protein- based Surface Engineering}

Intramolecular and intermolecular hydrogen bonds are essential elements sustaining 3-D structured domains in biomolecules, particularly in proteins and glycoproteins. Polar domains of proteins and polysaccharides bind water molecules to different degrees. As extensively documented in size exclusion chromatography and affinity chromatography assets, polysaccharides efficiently suppress non-specific adsorption [4]. Alternatively, there is extensive experimental evidence available demonstrating that albumin - a glycosylated serum protein - efficiently passivates surfaces and thus suppresses non-specific adsorption of analyte components. Enzyme-linked immuno sorbent assays, as performed in polystyrene wells may serve as example. Knowledge of these passivating properties of polysaccharides and proteins led to the use of proteins and polysaccharides as molecular polymeric units for probe immobilization [5]. Moreover, covalent and non-covalent assemblies of proteins with polysaccharides show improved long-term stability.

Multiple derivatization of polysaccharides and proteins such as dextran, hyaluronic acid or proteins like serum albumin with photoactive reagents yielded photolinker polymers that allow covalent binding of biomolecules, mixtures of biomolecules as well as low molecular weight substances to materials, simply by exposure to activating light. Dextran-based photolinker polymers are of particular interest in view of simultaneous stabilization of probe structures and surface passivation [6]. The resulting surface properties differ significantly from most of the current surface chemistries: dextran- or protein-based surfaces are hydrophilic and biocompatible. Their physico-chemical properties and chemical reactivities can be varied by judicious selection of the polymer base and the chemical functions. The Table lists a selection of dextran-based photolinker polymers (Opto Dex analogues) with different functional groups for secondary probe molecule bind- ing or facilitated detection. After photoimmobilization of the photolinker polymer to material surfaces, the secondary functional groups, e.g. amino groups, carboxy groups, activated thiol groups, biotin, become available for thermochemical or affinity binding.

\section{Functional Platforms for Capture Probe Immobilization}

Recently developed functional platforms provide advantageous characteristics for probe immobilization in microarray formats; some platforms offer the option of oriented biomolecule immobilization. Functional platforms can be based on microscope slide supports or other formats (microplates, microfluidic chips, etc). Dextran-based functionalized platforms present at the surface hydrogel-like thin-films. They are arranged in few molecular layers (ca. $10 \mathrm{ng} / \mathrm{mm}^{2}$ ). The homogeneity variation of OptoDex passivated surfaces is $<6 \%$ in a $20 \times 20 \mathrm{~mm}^{2}$ area. Such thin-film polysaccharide layers provide a biocompatible and stabilizing environment for most biomolecules, and effectively suppress non-specific binding. Specifications of the functional platforms have been established and several microarray applications have been investigated.

An application of a functional platform carrying carboxyl functions is demonstrated by covalent coupling of antigens, followed by immunostaining (Fig. 1). The antigens are locally immobilized by applying EDC/NHS chemistries after arraying onto chemically activated surfaces [7]. Antigen binding to the immobilized antigen is detected by binding of fluorescent-labeled antigen-specific antibodies exclu-

Table. Polysaccharide based functional platforms

Photochip platforms. The light-induced photoreaction enables covalent attachment of the linker polymer to material surfaces. After photobonding, the immobilized photolinker polymer provides chemical functional groups (amino-, carboxyl-, maleimido-, activated thiol- or biotin functions) that modulate the physical properties of the surface, and/or are available for chemical derivatization.

\begin{tabular}{|c|c|c|c|}
\hline Name & Surface coat ${ }^{a}$ & $\begin{array}{l}\text { Secondary } \\
\text { function/label }\end{array}$ & Coupling chemistry/physical property \\
\hline PhotoChip & OptoDex B & -Aryldiazirine & Light induced, carbene insertion \\
\hline Carboxy platform & OptoDex C & $-\mathrm{COOH}$ & $\mathrm{EDC} / \mathrm{NHS}^{\mathrm{b}}$ \\
\hline Amino platform & OptoDex A & $-\mathrm{NH} 2$ & Nucleophile substitution \\
\hline Thiol platform & OptoDex S & - S-S-aryl & Disulfide exchange \\
\hline Biotin platform & OptoDex Biotin & - Biotin & Biotin/avidin (streptavidin, neutravidin) \\
\hline Maleimide platform & OptoDex M & - Maleimide & Thiol (nucleophile) coupling \\
\hline Lactose platform & OpotDex Lac & - tethered lactose & Branched glycosylation \\
\hline Fluorescent platform & OptoDex Cy5 & - Cyanine dye: Cy5 & Fluorescence $\lambda_{\text {ex }} 649 \mathrm{~nm} ; \lambda_{\text {em }} 670 \mathrm{~nm}$ \\
\hline Fluorescent platform & OptoDex Cy3 & - Cyanine dye: Cy3 & Fluorescence $\lambda_{\mathrm{ex}} 550 \mathrm{~nm} ; \lambda_{\mathrm{em}}^{\mathrm{em}} 570 \mathrm{~nm}$ \\
\hline
\end{tabular}


Fig. 1. Immunostained protein array. Microarrayed antigens and buffer references (columns 1-4: mouse antigen; column 5: phosphate buffered saline (PBS buffer): columns 6-9: horse radish peroxidase (HRP) labeled antibody/bovine serum albumin (BSA); column 10: PBS buffer; columns 11-12: human antigen; columns 13-14: BSA) are immobilized on an OptoDex $\mathrm{C}$ coated glass platform (slide format) applying carbodiimide chemistries [7]. Coupled antigens are detected by confocal scanning for immunofluorescence after complexation with antimouse immunoglobulin-Cy5 conjugate and anti-HRP-Cy3 conjugate, respectively ( $A$ and B). C: fluorescence image and cross-section scan of immunostained protein spots on the carboxy-functionalized platform OptoDex C.

sively to the respective antigen. Negative controls including a human antigen and bovine serum albumin reveal low fluorescence signals supporting highly efficient suppression of non-specific binding (interaction specificity and background).

Stepwise affinity binding of neutravidin and a biotinylated-antigen to OptoDex Biotin platforms, and subsequent interaction with a fluorescent-labeled antibody indicated that Biotin OptoDex platforms enable avidin-mediated probe immobilization (Fig. 2). This regime is applicable to all probe molecules that are available as biotinylated and functionally bioactive conjugates (e.g. biotinylated proteins, biotinylated oligo- and polysaccharides, biotinylated oligonucleotides or DNA probes).

Fig. 3 shows a unique and conceptually different way to immobilize probe biomolecules on material surfaces. In this approach, the photolinker polymer is merely thin-film coated on the supporting substrate, and probe molecules are printed on the polymer layer before light exposure. Binding of the linker polymer to the surface

Fig. 2. Avidin-mediated biotinylated probe immobilization on OptoDex Biotin. A: OptoDex Biotin is printed and photobonded onto glass slides (columns 1-6: OptoDex Biotin; column 7: PBS buffer; columns 8-9: OptoDex B; columns 10-11: Cy5-OptoDex), and the presence of biotin is detected by binding of Cy $5 \mathrm{la}$ beled streptavidin. The intra-batch coefficient of variation (240 spots) is below $10 \%$. B: Photobonded OptoDex Biotin is detected on the surface by first binding neutravidin / biotinylated-mouse immunoglobulin, the latter being detected after immunocomplexation with Cy3-anti-mouse immunoglobulin.

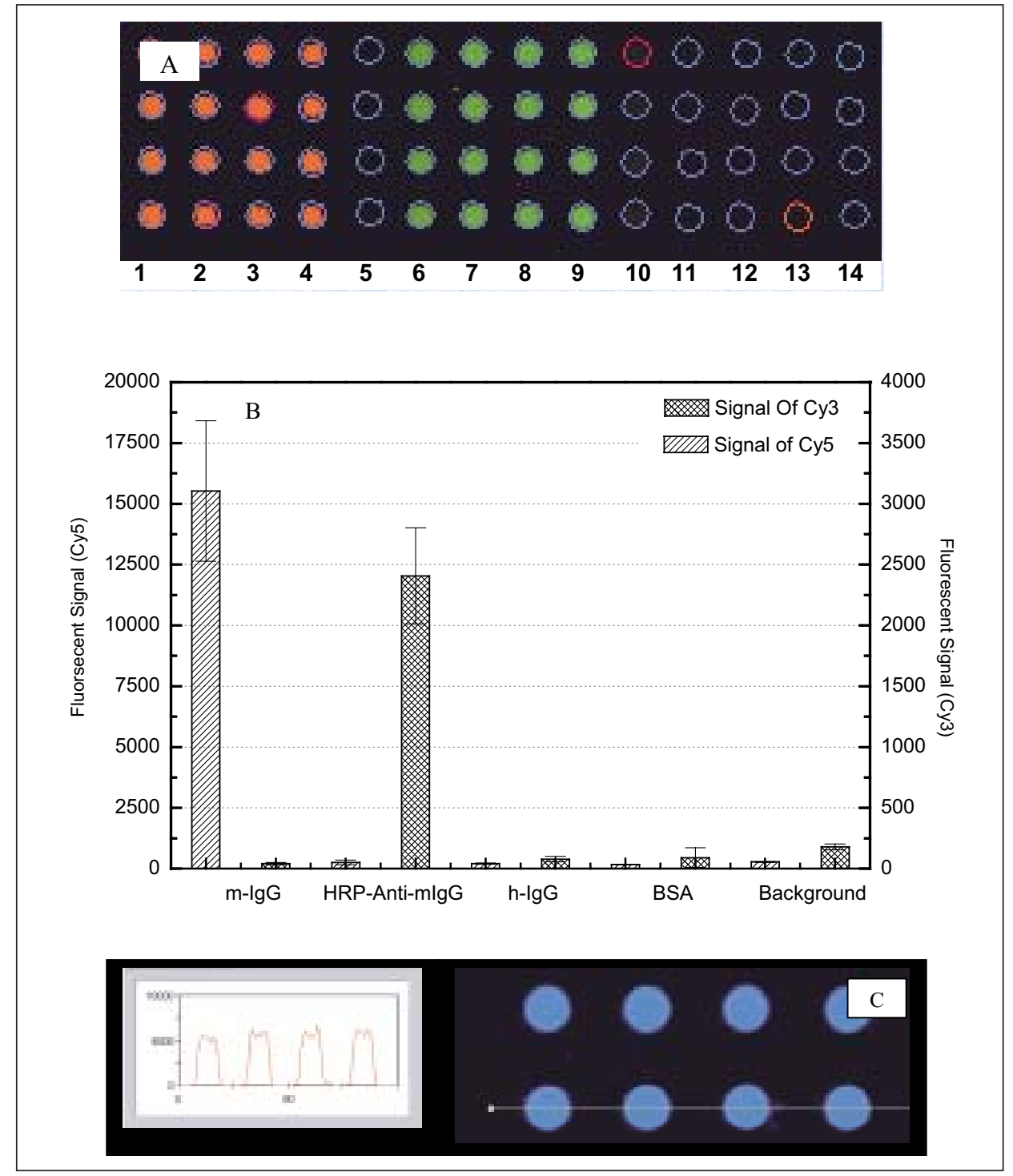

$\mathbf{A}$
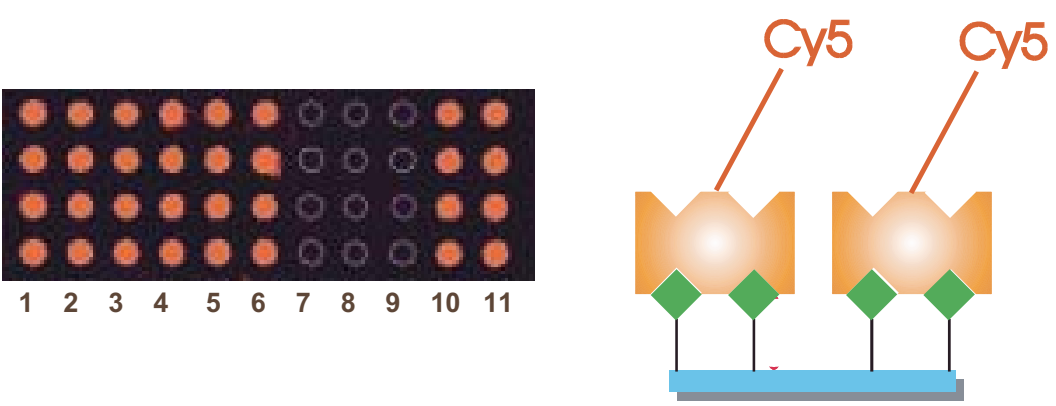

B

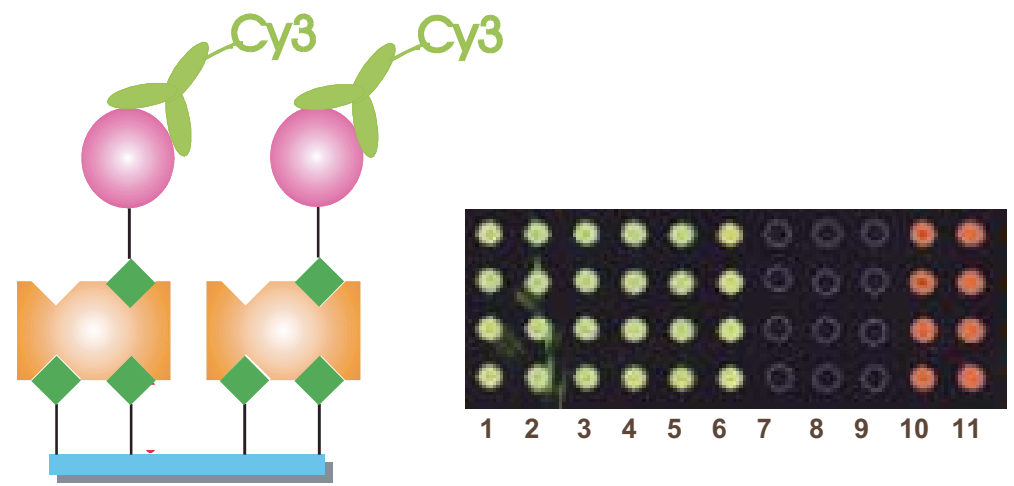


and binding of the probe molecule to the multiple photolinker derivatized polymer occurs simultaneously by irradiation with light (the photoactivation process is detailed in [6]). This process is favorably used for bioarray production purposes, providing the advantages of few process steps. Probe immobilization does not involve thermochemical chemistries. The results obtained with an array of antigens and subsequent immunostaining of photoimmobilized antigens are depicted in Fig. 3. Again, the probe molecules are immobilized on, and within the dextran base polymer, which leads to efficient suppression of non-specific binding. This last aspect was additionally confirmed by treatment of an antigen array with fluorescent $(\mathrm{Cy} 3)$ labeled serum albumin. Due to the presence of the OptoDex layer, the interaction of serum albumin with the array surface was insignificant (data not shown).

\section{Conclusion}

Photolinker polymer coated surfaces carrying secondary functional groups are unique tools for systems biology investigations, including genomics, proteomics, glycomics, metabolomics and cellomics. Considering both the biological requirements of probe molecules and device engineering, there are definite advantages and attractive commercial prospects: the fast and simple photobonding process leads to linker polymer binding and covalent passivation of any type of 'inert' surface including glass, polymer, metal oxide and many others, while providing a selection of chemical functions for probe molecule immobilization. Bioengineered platforms are applicable for fast molecular screening, for quality survey and control, and for efficient prognostic and diagnostic medical testing.

\section{Acknowledgement}

The Swiss Commission of Technology and Innovation in part supported the work presented. Financial support of the Albert Köchlin Foundation is gratefully acknowledged.

Received: July 14, 2003

[1] K.K. Jain, 'Molecular Diagnostics: Technologies, Markets and Companies', JainPharmaBiotech Publications, Basel, Switzerland 2003.

[2] S.P.A. Fodor, J.L. Read, M.C. Pirning, L. Stryer, A.T. Lu, D. Solas, Science 1991, 251,767

[3] G. MacBeath, S.L. Schreiber, Science 2000, 289, 1760.

[4] L. Hagel, H. Lundström, T. Andersson, $J$. Chromatogr. 1989, 476, 329.

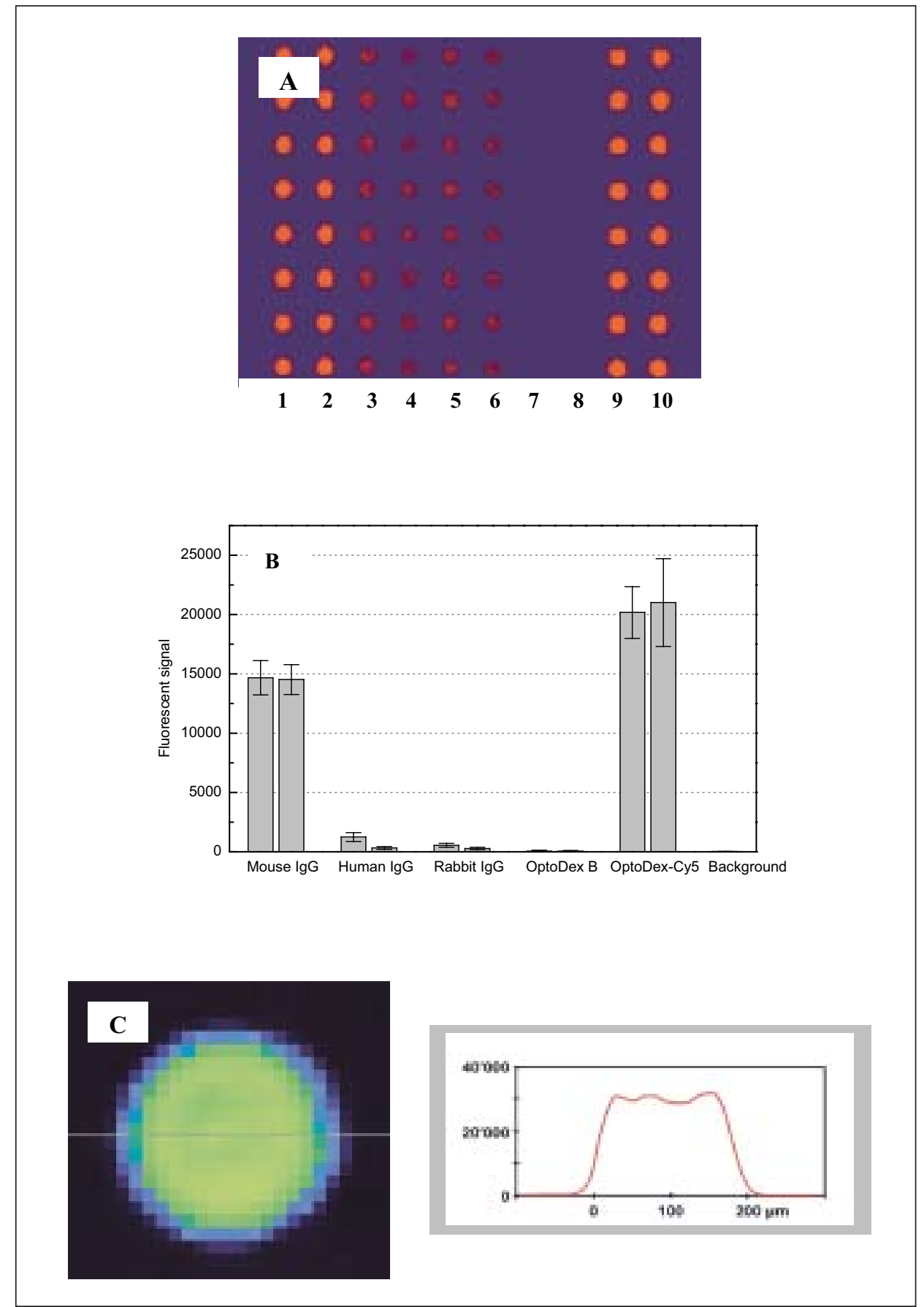

Fig. 3. Photobonded antigen array on a OptoDex B PhotoChip immunostained with fluorophore labeled anti-mouse immunoglobulin. A: columns 1-2: mouse antigen; columns 3-4: human antigen; columns 5-6: rabbit antigen; columns 7-8: OptoDex B; columns 9-10: Cy5-OptoDex. Immuno-complexation is detected by confocal scanning of Cy5-labeled anti-mouse immunoglobulin. B: Quantitative evaluation of the recovered fluorescence. C: Magnified photobonded and immunostained spot and fluorescence density (linescan). The diameter of Pin-and-Ring printed spots (50 pl deposited) on thin film OptoDex is 150 to $200 \mu \mathrm{m}$.

[5] H. Gao, E. Kislig, N. Oranth, H. Sigrist, Biotechnol. Appl. Biochem. 1994, 20,251; N. Barié, M. Rapp, H. Sigrist, H.J. Ache, Biosensors and Bioelectronics 1998, 13, 855.

[6] I. Caelen, H. Gao, H. Sigrist, Langmuir 2002, 19, 48; H. Sigrist, A. Collioud, J.F. Clémence, H. Gao, R. Luginbühl, M. Sänger, G. Sundarababu, Opt. Eng. 1995, 34, 2339.

[7] Functionalization of a OptoDex C platform. Immediately prior to use, equal volumes of NHS (N-hydroxysuccinimide,
$100 \mathrm{mM}$ ) and EDC (1-ethyl-3-(3-dimethylaminopropyl) carbodiimide, 400 $\mathrm{mM}$ ) were mixed and applied on the OptoDex $\mathrm{C}$ platform. After incubating the surface for $1 \mathrm{~h}$ at room temperature, the EDC/NHS mixture is removed, rinsed with sodium acetate buffer $(10 \mathrm{mM}, \mathrm{pH}$, 5.0). Antigens were then arrayed on the activated platforms and after rinsing of the surfaces, specific immunointeractions were detected by binding of fluorescently labeled antigen-specific antibodies. 